\title{
Cell Microscopic Segmentation with Spiking Neuron Networks
}

\author{
Boudjelal Meftah ${ }^{1,2}$, Olivier Lezoray ${ }^{2}$, \\ Michel Lecluse ${ }^{3}$, and Abdelkader Benyettou ${ }^{4}$ \\ 1 Equipe EDTEC, Université de Mascara, Mascara, Algérie \\ 2 Université de Caen Basse-Normandie, GREYC UMR CNRS 6072, \\ 6 Bd. Maréchal Juin, F-14050, Caen, France \\ 3 Service d'anatomie et de cytologie pathologiques, Centre Hospitalier Public du \\ Cotentin, Rue du Val de Saire, F-50130 Cherbourg-Octeville, France \\ 4 Laboratoire Signal Image et Parole, Université Mohamed Boudiaf, Oran, Algérie
}

\begin{abstract}
Spiking Neuron Networks (SNNs) overcome the computational power of neural networks made of thresholds or sigmoidal units. Indeed, SNNs add a new dimension, the temporal axis, to the representation capacity and the processing abilities of neural networks. In this paper, we present how SNN can be applied with efficacy for cell microscopic image segmentation. Results obtained confirm the validity of the approach. The strategy is performed on cytological color images. Quantitative measures are used to evaluate the resulting segmentations.
\end{abstract}

Keywords: Cell microscopic images, Hebbian learning, Segmentation, Spiking Neuron Networks.

\section{Introduction}

Image analysis in the field of cancer screening is a significant tool for cytopathology [1, [2]. Two principal reasons can be highlighted. First, the quantitative analysis of shape and structure of nuclei coming from microscopic color images brings to the pathologist valuable information for diagnosis assistance. Second, the quantity of information that the pathologist must deal with is large, in particular when the number of cancer screening increases. That is why, a segmentation scheme for microscopic cellular imaging must be efficient for reliable analysis.

Many cellular segmentation methods have been presented so far [3, 4]. They include watershed [5, [6], 7], region-based [ $[$ ] and threshold-based methods [9]. Application of active contour has been widely investigated for cell segmentation [10, 11]. Cells stained with Papanicolaou international staining make it possible to classify the color pixels among three classes [12]: background, cytoplasm or nucleus. However, this classification cannot be perfect. Indeed, a fraction on nuclei pixels have the same color then cytoplasm pixels because of the variability of the nuclei according to the type of the cells and to the chromatin distribution. Moreover, for some cytopathologies, the mucus present in the background has the same color than some cells (cytoplasm and nucleus). 
Another problem for the design of cellular segmentation schemes is on how to evaluate the segmentation quality. Indeed, almost all the segmentation schemes have some parameters. Human observation highlights that the values chosen for these parameters are significant for the quality of the segmentation. However, for an automatic selection of the optimal parameter values, the quality of segmentation must be also automatically evaluated. In literature, there are several quality segmentation criteria: Lui and Borsotti [13, classification rates and other statistical measures [14.

Spiking Neuron Networks (SNNs) are often referred to as the $3^{\text {rd }}$ generation of neural networks [15. Highly inspired from natural computing in the brain and recent advances in neuroscience, they derive their strength and interest from an accurate modeling of synaptic interactions between neurons, taking into account the time of spike firing. SNNs overcome the computational power of neural networks made of thresholds or sigmoidal units [16. The use of spiking neurons promises high relevance for biological systems and, furthermore, might be more flexible for computer vision applications [17.

In this paper, a spiking neural network is used to segment cellular microscopic images with two approaches: unsupervised and supervised training with Hebbian based winner-take-all learning. This learning modifies the weights of the presynaptic neurons with the winning output [18. This observation is in agreement with the fact that, in biological neural networks, different axonal connections will have different signal transmission delays 19. In this article, we seek, through a series of experiments, the best parameters of the SNN network to have a good segmentation.

The paper is organized as follows : in the first Section, related works are presented within the literature of spiking neural network (SNNs). Second Section is the central part of the paper and is devoted to the description of the architecture of a spiking neural network with multiple delay connections, the encoding mechanism for converting the real valued inputs into time vectors and the learning rule. Results and discussions of the experiments are reported in the third Section. Last Section concludes.

\section{Spiking Neuron Networks}

Spiking neural networks (SNNs) are a class of ANNs that are increasingly receiving the attention as both a computationally powerful and biologically plausible mode of computation 20, 21. SNNs model the precise time of the spikes fired by a neuron, as opposed to the conventional neural networks which model only the average firing rate of the neurons. It is proved that the neurons that convey information by individual spike times are computationally more powerful than the neurons with sigmoidal activation functions 22.

A network architecture consists in a feedforward network of spiking neurons with multiple delayed synaptic terminals (Fig:1(a)). Neurons in the network generate action potentials, or spikes, when the internal neuron state variable, called "membrane potential", crosses a threshold $\vartheta$. The relationship between input 
spikes and the internal state variable is described by the spike response model (SRM), as introduced by Gerstner [23. Depending on the choice of suitable spike-response functions, one can adapt this model to reflect the dynamics of a large variety of different spiking neurons. Formally, a neuron $j$, having a set

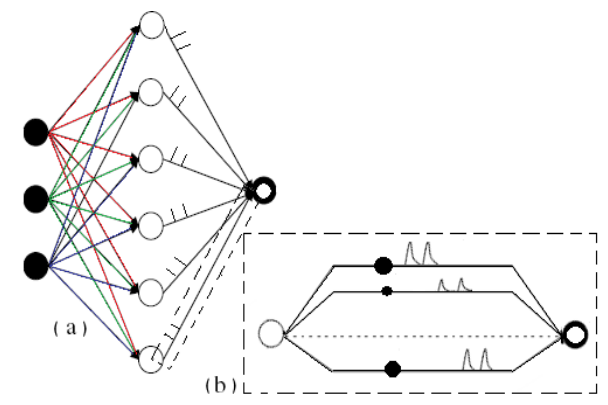

Fig. 1. (a) Spiking neural network architecture; (b) Multiple synapses transmitting multiple spikes

$\Gamma_{j}$ of immediate predecessors ("pre-synaptic neurons"), receives a set of spikes with firing times $t_{i}, i \in \Gamma_{j}$. Any neuron generates at most one spike during the simulation interval, and fires when the internal state variable reaches a threshold $\vartheta$. The dynamics of the internal state variable $x_{j}(t)$ are determined by the impinging spikes, whose impact is described by the spike-response function $\varepsilon(t)$ modeling a simple $\alpha$-function weighted by the synaptic efficacy $w_{i j}$ :

$$
x_{j}(t)=\sum_{i \in \Gamma_{j}} \sum_{k=1}^{m} w_{i j}^{k} \varepsilon\left(t-t_{i}-d^{k}\right)
$$

In the network as introduced in 24], an individual connection consists in a fixed number of $m$ synaptic terminals, where each terminal serves as a sub-connection that is associated with a different delay and weight (Fig 1(b)). The delay $d^{k}$ of a synaptic terminal $k$ is defined by the difference between the firing time of the pre-synaptic neuron, and the time the post-synaptic potential starts rising.

\section{Network Architecture, Learning and Encoding}

However, before building a SNN, we have to explore three important issues: network architecture, information encoding and learning method. Then, we will use a SNN to segment cellular images.

\subsection{Network Architecture}

The network architecture consists in a fully connected feedforward network of spiking neurons with connections implemented as multiple delayed synaptic terminals. We consider two different topologies for unsupervised and supervised 
learning. For unsupervised learning, the SNN performs its learning directly on the pixels of the image to classify. For unsupervised learning, a reference data set of pixels from different images is used for learning.

In both topologies depicted in Figure 2(a) and Figure 2(b), the network consists in an input layer, a hidden layer, and an output layer. The first layer is composed of RGB values of pixels. Each node in the hidden layer has a localized activation $\Phi^{n}=\Phi\left(\left\|X-C_{n}\right\|, \sigma_{n}\right)$ where $\Phi^{n}($.$) is a radial basis function (RBF)$ localized around $C_{n}$ with the degree of localization parameterized by $\sigma_{n}$. Choosing $\Phi(Z, \sigma)=\exp -\left(Z^{2} / 2 \sigma^{2}\right)$ gives the Gaussian RBF. This layer transforms the RGB values of pixels in first layer to temporal values. Third layer consist in class outputs (cell background, cytoplasm and nuclei).

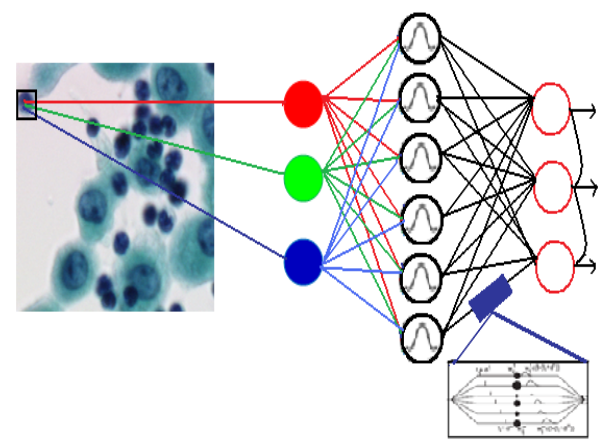

(a)

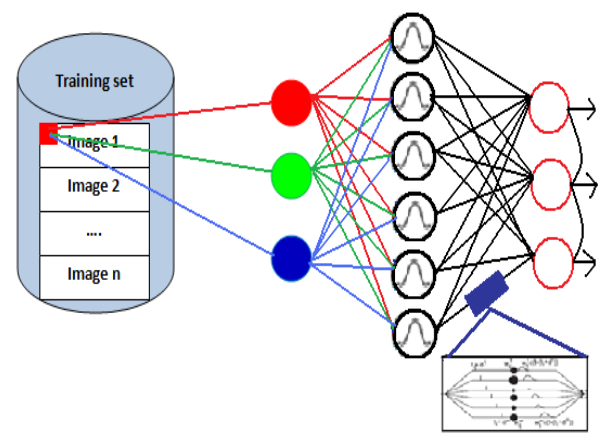

(b)

Fig. 2. (a) Network topology for unsupervised training; (b) Network topology for supervised training

Instead of a single synapse, with its specific delay and weight, this synapse model consists in many sub-synapses, each one with its own weight and delay $d^{k}$, as shown in Figure 1, b. The total contribution of all presynaptic neurons is given by equation (11). The neuron model implemented is the $S R M_{0}$ [23]. The delays $d^{k}$ are fixed for all sub-synapses $k$, varying from zero in $1 \mathrm{~ms}$ fixed intervals. 


\subsection{Information Encoding}

Bohte et al. [25], presented a method for encoding the input data to enhance the precision. Each neuron of entry is modeled by a local receiving field (RF). For a variable with range of [Max..Min], a set of $m$ Gaussian receptive RF neurons are used. The center $C_{i}$ and the width $\sigma_{i}$ of each RF neuron i are determined by the following equations:

$$
\begin{gathered}
C_{i}=I_{\min }+\left(\frac{2 i-3}{2}\right)\left(\frac{I_{\max }-I_{\min }}{m-2}\right) \\
\sigma_{i}=\frac{1}{\gamma} \frac{I_{\max }-I_{\min }}{m-2}
\end{gathered}
$$

where $m$ is number of receptive fields in each population and a value of 1.5 is used for the variable $\gamma$. For each $n$-dimensional input pattern, the encoding scheme results in a matrix $n \times m$ of values between 0 and 1 . These values are then converted to delay times. While converting the activation values of RFs into firing times, a threshold has been imposed on the activation value. A receptive field that gives an activation value less than this threshold will be marked as not-firing and the corresponding input neuron will not contribute to the postsynaptic potential.

\subsection{Learning Method}

The approach presented here implements the Hebbian reinforcement learning method through a winner-take-all algorithm [26, 27]. For unsupervised learning, a Winner-Takes-All learning rule modifies the weights between the input neurons and the neuron first to fire in the output layer using a time-variant of Hebbian learning. The synaptic weights should be randomly initialized. When an input pattern is presented to the network, neurons are expected to fire. The first neuron to fire is called the winner of the competitive process. Only the weights of the winner neuron are updated using a Hebbian learning rule $L(\Delta t)$. This learning function controls the learning process by updating the weight. It increases the weights of the connections that received spikes immediately before the fire of $j$ and decrease remaining weights. For a weight with delay $d^{k}$ from neuron $i$ to neuron $j$ we use [28]:

$$
\Delta w_{i j}^{k}=\eta L\left(\Delta t_{i j}\right)
$$

And

$$
L(\Delta t)=(1+b) e^{\frac{(\Delta t-c)^{2}}{2(k-1)}}-b
$$

with

$$
k=1-\frac{\nu^{2}}{2 \ln \frac{b}{1+b}}
$$

where: $\mathrm{L}($.$) is the learning function; \eta$ is the learning rate; $\nu$ determines the width of the learning window; $\Delta t$ is the difference between the arriving of the spike 
and the fire of neuron $j ; b$ determines the negative update given to a neuron; $c$ fixes the peak of the learning function; $w_{i j}^{k}$ is the increase of the $k^{\text {th }}$ connection between neurons $i$ and $j$. The weights are limited to the range 0 to $w_{\max }$, the maximum value that a weight can take.

\section{Experimental Results and Discussion}

\subsection{Microscopic Cells Database}

For the considered class of microscopic images, a microscopy expert has to choose judicious images that well describe the whole segmentation problem (a ground truth). This ground truth database can be used for the learning step and also as a reference segmentation to evaluate the relevance of an automatic segmentation. In the sequel, we will consider a publicly available database ${ }^{1}$ [29] of 8 microscopic images of bronchial tumors (752 x 574 pixels). The pixels of these images have to be classified into one of the three following classes background, cell cytoplasm and cell nucleus. Figure 3 (a)-(b) shows a microscopic color image and its ground truth. Pixel dataset has been split to produce training, validation and test sets.

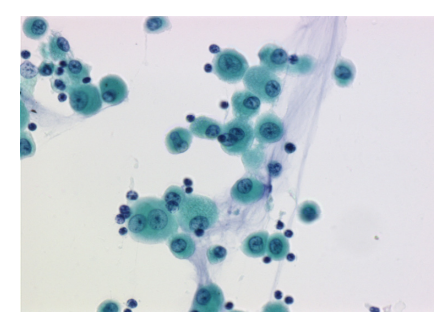

(a)

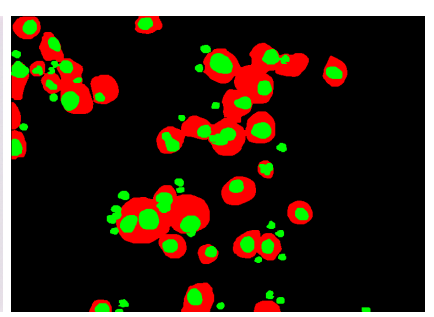

(b)

Fig. 3. (a) Original image; (b) Ground truth

\subsection{Segmentation Results}

Several experiments are carried out by changing the number of synapses, the number of receptive fields and the size of training corpus to select the best network parameters. Table 1 show these parameters.

Table 1. Best parameter of the SNN

\begin{tabular}{|l|l|l|l|l|l|l|l|l|}
\hline $\begin{array}{l}\text { Receptive } \\
\text { field }\end{array}$ & Subsynapse & Threshold & $\begin{array}{l}\text { Training } \\
\text { set }\end{array}$ & $\eta$ & $\tau$ & $v$ & b & c \\
\hline 8 & 12 & 9 & $10 \%$ & 0.0025 & 3 & 5 & -0.007 & -2.3 \\
\hline
\end{tabular}

\footnotetext{
${ }^{1}$ http://users.info.unicaen.fr/ lezoray/database.php
} 

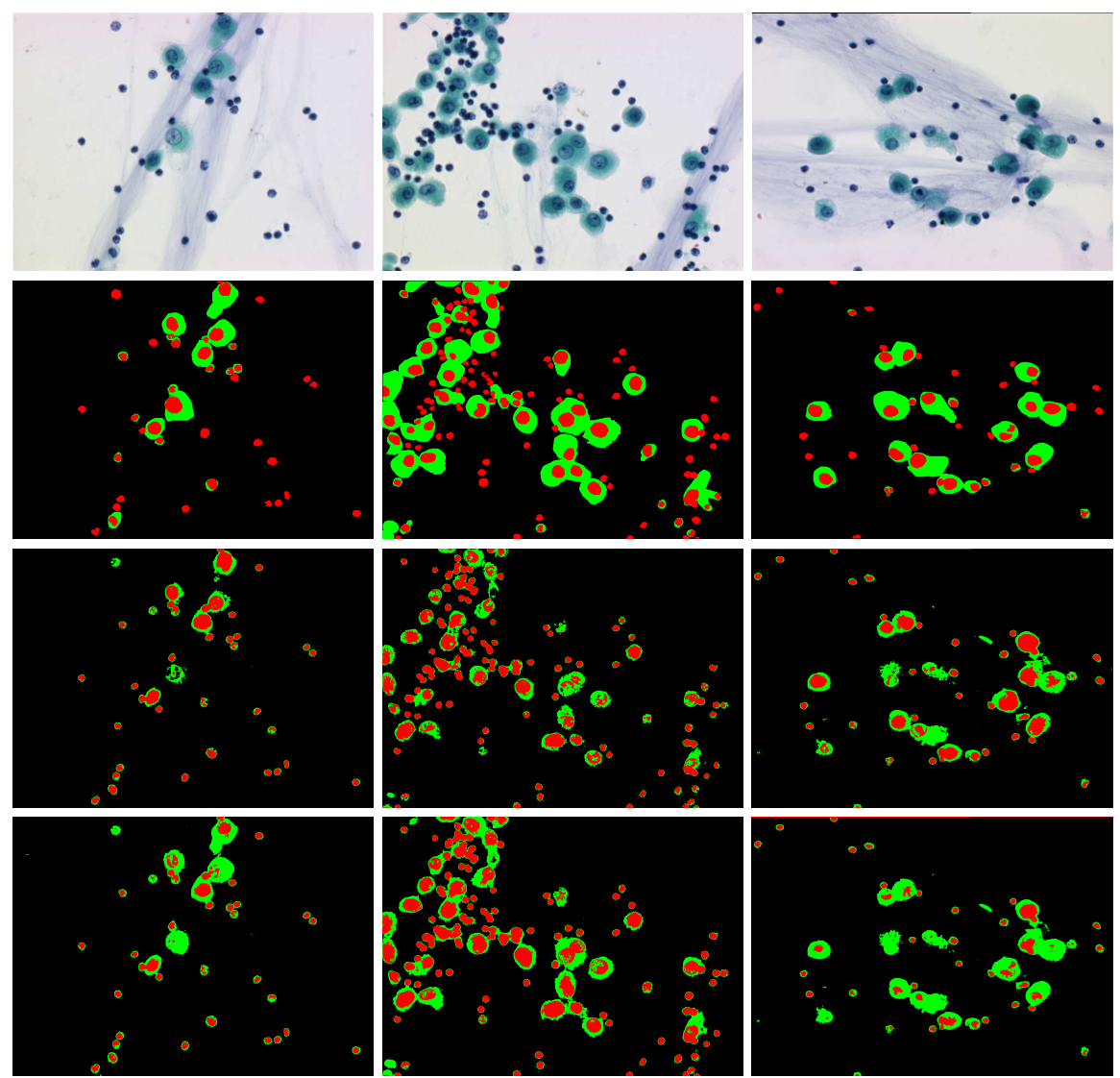

Fig. 4. Cell microscopic images (First row); expert segmentation (Second row); segmentation produced by unsupervised training (Third row) and segmentation produced by supervised training (Fourth row)

Images in Figure 4 show segmentation results with our segmentation scheme in comparison with the expert segmentation. It is worth to note that the mucus present in all images is correctly identified as background.

\subsection{Evaluation Methods}

To evaluate our approach, we use several classification rates. These classifications rates are expressed as follows:

$$
\begin{aligned}
& R_{0}=\frac{\text { Number of pixels well classified }}{\text { Number of pixels of the image }} \\
& R_{1}=\frac{\text { Number of nuclei pixels well classified }}{\text { Number of nuclei pixels of the image }} \\
& R_{2}=\frac{\text { Number of background pixels well classified }}{\text { Number of background pixels of the image }} \\
& R_{3}=\frac{R_{1}+R_{2}}{2}
\end{aligned}
$$


Results in Table 2 show that SNN with supervised training has the best classification accuracies as compared to SNN with unsupervised training.

Table 2. Classification rates (best rates bold faced)

\begin{tabular}{|l|l|l|}
\hline & $\begin{array}{l}\text { SNN with unsupervised } \\
\text { training }\end{array}$ & $\begin{array}{l}\text { SNN with supervised train- } \\
\text { ing }\end{array}$ \\
\hline$R_{0}$ & $89.07 \%$ & $\mathbf{9 4 . 2 7} \%$ \\
\hline$R_{1}$ & $69.57 \%$ & $\mathbf{8 0 . 3 7} \%$ \\
\hline$R_{2}$ & $94.55 \%$ & $\mathbf{9 9 . 0 6} \%$ \\
\hline$R_{3}$ & $82.06 \%$ & $\mathbf{8 9 . 7 1} \%$ \\
\hline
\end{tabular}

Table 3 presents a comparison of the the classification accuracies obtained by Meurie et al. 29] for different classifiers as well as with our SNN supervised training. Our approach clearly outperforms all these state-of-the-art methods.

Table 3. Segmentation rates and comparison with Meurie et al. approaches [29, with best rates bold faced

\begin{tabular}{|l|l|}
\hline Classifier & $R_{1}$ \\
\hline SVM & $74.2 \%$ \\
\hline Bayes & $74.6 \%$ \\
\hline K-means & $74.4 \%$ \\
\hline MLP & $73 \%$ \\
\hline Fisher 1 & $72.3 \%$ \\
\hline KNN & $70 \%$ \\
\hline Supervised SNN & $\mathbf{8 0 . 3 7 \%}$ \\
\hline
\end{tabular}

\section{Conclusion}

An automated approach for the segmentation of cells has been presented. Segmentation is based on spiking neural networks with unsupervised training and supervised training. At first, the network is build, a subset of the images pixels is taken to be learned by the network and finally the SNN processes the rest of the images to have as a result a number of classes quantizing the cell image.

\section{References}

1. Knesek Jr., E.A.: Roche Image Analysis System. Acta Cytologica 40(1), 60-66 (1996)

2. Patten Jr., S.F., Lee, S.S.J., Nelson, A.C.: Neopath Autopap 300 Automatic pap Screener System. Acta Cytologica 40(1), 45-52 (1996) 
3. Di Rubeto, C., Dempster, A., Khan, S., Jarra, B.: Segmentation of Blood Image using Morphological Operators. In: Proc. of the 15th Int. Conference on Pattern Recognition, Barcelona, Spain, September 3-8, vol. 3, pp. 397-400 (2000)

4. Anoraganingrum, D.: Cell Segmentation with Median Filter and Mathematical Morphology Operation. In: Proc. of the International Conference on Image Analysis and Processing, Venice, Italy, September 27-29, pp. 1043-1046 (1999)

5. Lezoray, O., Cardot, H.: Cooperation of Pixel Classification Schemes and Color Watershed: a Study for Microscopical Images. IEEE Transactions on Images Processing 11(7), 738-789 (2002)

6. Lin, G., Adiga, U., Olson, K., Guzowski, J.F., Barnes, C.A., Roysam, B.: A hybrid 3D Watershed Algorithm Incorporating Gradient Cues and Object Models for Automatic Segmentation of Nuclei in Confocal Image Stacks. Cytometry A 56(1), 23-36 (2003)

7. Adiga, U.P.S., Chaudhuri, B.B.: An Efficient Method based on Watershed and Rulebased Merging for Segmentation of 3-D Histopathological Images. Pattern Recognition 34(7), 1449-1458 (2001)

8. Mouroutis, T., Roberts, S.J., Bharath, A.A.: Robust Cell Nuclei Segmentation Using Statistical Modeling. BioImaging 6, 79-91 (1998)

9. Wu, H.S., Barba, J., Gil, J.: Iterative Thresholding for Segmentation of Cells from Noisy Images. J. Microsc. 197, 296-304 (2000)

10. Karlosson, A., Strahlen, K., Heyden, A.: Segmentation of Histological Section using Snakes. In: Bigun, J., Gustavsson, T. (eds.) SCIA 2003. LNCS, vol. 2749, pp. 595602. Springer, Heidelberg (2003)

11. Murashov, D.: Two-Level Method for Segmentation of Cytological Images using Active Contour Model. In: Proc. of the 7th Int. Conference on Pattern Recognition and Image Analysis, PRIA-7, St. Petersburg, Russian Federation, October 18-23, vol. III, pp. 814-817 (2004)

12. Papanicolaou, G.N.: A new procedure for staining vaginal smears. Science 95,432 (1942)

13. Borsotti, M., Campadelli, P., Schettini, R.: Quantitative Evaluation of Color Image Segmentation Results. Pattern Recognition Letters 19, 741-747 (1998)

14. Glory, E., Meas-Yedid, V., Pinset, C., Olivo-Marin, J.C., Stamon, G.: A Quantitative Criterion to Evaluate Color Segmentation Application to Cytological Imges. In: Blanc-Talon, J., Philips, W., Popescu, D.C., Scheunders, P. (eds.) ACIVS 2005. LNCS, vol. 3708, pp. 227-234. Springer, Heidelberg (2005)

15. Maass, W.: Networks of spiking neurons: The third generation of neural network models. Neural Networks 10(9), 1659-1671 (1997)

16. Paugam-Moisy, H., Bohte, S.M.: Computing with Spiking Neuron Networks. In: Kok, J., Heskes, T. (eds.) Handbook of Natural Computing, 40p. Springer, Heidelberg (2009) (to appear)

17. Thorpe, S.J., Delorme, A., VanRullen, R.: Spike-based strategies for rapid processing. Neural Networks 14(6-7), 715-726 (2001)

18. Gupta, A., Long, L.N.: Hebbian learning with winner take all for spiking neural networks. In: IEEE International Joint Conference on Neural Networks (IJCNN), Atlanta, Gerogia, pp. 1189-1195 (2009)

19. Wu, B.J.: Introduction to neural dynamics and signal transmission delay. Walther de Gruyter, Berlin (2001)

20. Maass, W.: On the relevance neural networks. MIT-Press, London (2001)

21. Gerstner, W., Kistler, W.M.: Spiking neuron models, 1st edn. Cambridge University Press, Cambridge (2002) 
22. Maass, W.: Fast sigmoidal networks via spiking neurons. Neural Computation 9, 279-304 (1997)

23. Gerstner, W.: Time structure of the activity in neural network models. Phys. Rev. E 51, 738-758 (1995)

24. NatschlNager, T., Ruf, B.: Spatial and Temporal Pattern Analysis via Spiking Neurons Network. Comp. Neural Systems 9(3), 319-332 (1998)

25. Bohte, S.M., La Poutre, H., Kok, J.N.: Unsupervised clustering with spiking neurons by sparse temporal coding and Multi-Layer RBF Networks. IEEE Transactions on Neural Networks 13(2), 426-435 (2002)

26. Ludemir, B.T.B., De Carvalho, C.P.: Artificial neural networks - theory and applications, 1st edn. LTC Editora, Rio de Janeiro (2000)

27. Oster, M., Liu, S.C.: A winner-take-all spiking network with spiking inputs. In: Proceedings of the 11th IEEE International Conference on Electronics, Circuits and Systems (ICECS 2004), vol. 11, pp. 203-206 (2004)

28. De Berredo, R.C.: A review of spiking neuron models and applications. M. Sc. Dissertation, University of Minas Gerais (2005)

29. Meurie, C., Lezoray, O., Carrier, C., Elmoataz, A.: Combination of Multiple Pixel Classifiers for Microscopic Image Segmentation. International Jornal of Robotic and Automation, Special Issue on Color Image and Analysis for Machine Vision 20(2), 63-69 (2005) 